\section{Hilft Vitamin C wirklich gegen Schnupfen?}

Viele Menschen schwören auf Vitamin C zur Prophylaxe und Therapie des Schnupfens. Ob diese Methode Sinn macht, untersuchten Wissenschaftler in einer aktuellen Studie.

sraelische Forscher randomisierten 39 Jugendliche, die regelmäßig für Schwimmwettkämpfe trainierten. Die Probanden erhielten für drei Monate täglich entweder $1 \mathrm{~g}$ Vitamin C oder Placebo.

Die Häufigkeit der in diesem Zeitraum auftretenden Erkältungskrankheiten wur-

de durch diese Prophylaxe nicht reduziert. Auch die Länge des Schnupfens war im Durchschnitt nicht signifikant kürzer. Allerdings ergab sich hier ein nicht signifikanter positiver Trend, der für die Vitamin-C-Gabe sprach. Weitere Analysen deuteten ebenfalls eine Wirksamkeit der

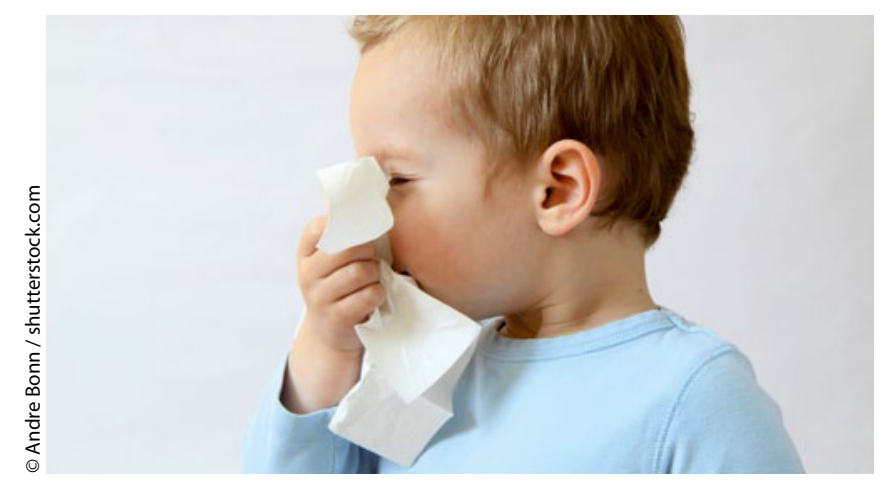

Dauer von Erkältungskrankheiten durch Vitamin C gesenkt - zumindest ein positiver Trend ist nachweisbar.
Ascorbinsäure an. In der Subgruppe der männlichen Studienteilnehmer waren die schnupfenverkürzenden Effekte sogar signifikant. Die Autoren meinen, dass diese Ergebnisse erfolgversprechend sind und weiterer Forschung bedürfen.

Constantini NW et al. The effect of vitamin C on upper respiratory infections in adolescent swimmers: a randomized trial. Eur J Pediatr 2011; 170: 59-63

Kommentar: Inzwischen gibt es zahlreiche Studien zum Thema Schnupfenprophylaxe mit Vitamin C. Einige von ihnen scheinen das zu bestätigen, was die hier vorliegende, leider sehr kleine Studie andeutet: Die Erkältungsdauer kann durch regelmäßige Einnahme von hoch dosiertem Vitamin C verkürzt werden. Der Effekt ist jedoch nur marginal. Daher sind Studien mit niedrigen Fallzahlen auch wenig geeignet, uns hier erheblich weiterzuhelfen. Fazit: Vitamin C verhütet einen Schnupfen wohl nicht, kann ihn aber (um einige Stunden) abkürzen.

Prof. Dr. Edzard Ernst

\title{
Regulationsstörungen: Assoziation mit späteren Auffälligkeiten
}

Rund 20\% aller Kinder leiden an exzessiven Regulationsstörungen - unter ihnen leiden nicht unerheblich auch ihre Eltern. Schweizer Autoren stellen eine Metaanalyse vor, die die Assoziation von frühkindlichen Regulationsstörungen mit Verhaltensstörungen in der Kindheit unter Beweis stellt.

$\mathrm{n}$ die Analyse gingen 22 Studien ein, die den Anforderungen der Autoren entsprachen: Regulationsstörungen im ersten Lebensjahr, Untersuchung von internalisierendem sowie externalisierendem Verhalten, ADHS oder generelle Verhaltensauffälligkeiten in der Kindheit mit statistischen Angaben sowie prospektives Studiendesign. 16.848 Kinder mit Regulationsstörungen wurden erfasst.

Es bestand eine signifikante Effektstärke für die Assoziation Schreistörung und externalisierende Störungen sowie eine etwas schwächere Effektstärke für ADHS und internalisierende Störungen. Fütterstörungen korrelierten nur sehr schwach mit Verhaltensstörungen; Schlafstörungen korrelierten stark mit ADHS und schwach mit internalisierenden und generellen
Verhaltensproblemen. Das höchste Risiko für in der Kindheit auftretende Verhaltensstörungen bestand bei der Kombination von Regulationsstörungen und erhöhtem familiären Risiko.

Hemmi M et al. Associations between problems with crying, sleeping, and/or feeding in infancy and long term behavioural outcomes in childhood: a meta-analysis. Arch Dis Child 2011; 96: 622-9

Kommentar: Auch wenn Dreimonats-Koliken bei den meisten Säuglingen nur der Ausdruck von vorübergehenden Anpassungsstörungen ohne spätere negative Auswirkungen auf das Verhalten darstellten, so ist das bei exzessivem Schreien, Schlafund Fütterstörungen nicht immer der Fall.
Je mehr zusätzliche Belastungen für die Eltern bestehen, desto geringer wird deren Kompensationsfähigkeit sein. So ist nachvollziehbar, dass ungünstige psychosoziale familiäre Faktoren mit Verhaltensstörungen im Kindesalter stark korrelieren. Vorsicht ist jedoch geboten in der Erstellung der Kausalität - zu behaupten, dass die ungünstigen psychosozialen Faktoren die Ursache der Verhaltensstörungen sind, ist meist zu wenig differenziert gesehen. Vielmehr ist davon auszugehen, dass ungünstige soziale Verhältnisse einen Multiplikator für möglicherweise genetisch bedingte Vulnerabilitätsfaktoren darstellt und die Ausprägung der Störung verstärkt.

Regulationsstörungen sind als unspezifisches Syndrom zu sehen. Einer der Auslöser kann eine ADHS sein. Für die Praxis wichtig ist die Beobachtung, dass bei Kindern mit Regulationsstörungen das Risiko für Verhaltensstörungen unterschiedlichster Art erhöht ist - daher sollten diese Kinder engmaschiger kontrolliert werden, insbesondere wenn zusätzlich familiäre Risikofaktoren vorliegen. Hier sollte eventuell auch rechtzeitig das Jugendamt hinzugezogen werden.

Dr. Kisten Stollhoff 